\title{
Smoking habits among pregnant Danish women: reliability of information recorded after delivery
}

\author{
Ulrik Kesmodel, Sjúrdur Fródi Olsen
}

\begin{abstract}
Study objective-To compare recall of smoking habits during pregnancy 0.5-3 years after delivery across groups defined by recall time ( 5 six month periods) and pregnancy outcome (pre-eclampsia, pregnancy induced hypertension, intrauterine growth retardation, preterm or post-term delivery compared with controls). Design-Case-control nested in cohort study.

Setting and participants-A subsample of 503 women from a cohort of 6347 women established between 1989 and 1991 in Aarhus University Hospital.

Main results-Measures of agreement between concurrent and retrospective data on smoking status varied between 0.93 and 1.0 (sensitivity), 0.90 and 0.98 (specificity), and 0.79 and 0.98 (к). Spearman's correlation coefficients for number of cigarettes smoked/day varied between 0.87 and 0.97 ; mean differences were all close to zero. Accuracy of recall tended to diminish with increasing alcohol intake, particularly among women smoking $\geqslant 10$ cigarettes/day.

Conclusions-Information on smoking habits could be accurately obtained retrospectively independent of recall time and the pregnancy outcomes studied here. Accuracy diminished with increasing alcohol intake, particularly among heavy smokers.
\end{abstract}

(F Epidemiol Community Health 1999;53:239-242)

Perinatal

Epidemiological

Research Unit,

Department of

Obstetrics and

Gynaecology, Aarhus

University Hospital,

Aarhus, Denmark

U Kesmodel

Danish Epidemiology Science Centre, Statens Serum Institut, Copenhagen,

Denmark

S F Olsen

Correspondence to: Dr U Kesmodel, Perinatal Epidemiological Research Unit, Department of

Obstetrics and Gynaecology, Skejby Sygehus, Aarhus University Hospital, 8200 Aarhus N, Denmark.

Accepted for publication 6 October 1998
It is well documented that smoking in pregnancy is associated with reduced fetal growth, ${ }^{1}$ abruptio placentae and placenta previa, ${ }^{2}$ perinatal mortality, ${ }^{3}$ and other ill health. When the complications studied are rare, the case-control design with retrospective assessment of exposure offers a very cost effective epidemiological design. A major weakness inherent in this design, however, is that spurious associations may be created between such retrospectively collected exposure data and pregnancy outcome caused by deterioration of recall over time $^{4-6}$ and differential misclassification between cases and controls.

The extent to which such misclassification takes place when smoking in pregnancy is assessed after delivery has been studied under various conditions, but because of inconsistent results further exploration and documentation is warranted.

In Danish women in whom smoking habits in pregnancy had been assessed concurrently and retrospectively we attempted to examine to what extent recall depended on recall time and pregnancy outcome. Observed differences were also related to baseline variables such as social status and alcohol intake.

\section{Methods}

STUDY POPULATION AND SELECTION PROCEDURE Data used in this study were from an earlier published case-control nested in cohort study. ${ }^{8}$ Subjects were a subsample from a cohort of 9434 pregnant women established during an approximate two year period between 1989 and 1991 in Aarhus University Hospital, covering a well defined geographical area. Approximately $99 \%$ of women in the area comply with the antenatal care programme. The associations between certain dietary factors in pregnancy and the risk of pre-eclampsia, pregnancy induced hypertension (PIH), intrauterine growth retardation (IUGR), preterm and post-term delivery were examined ${ }^{8}$ by expanding the cohort data base by a questionnaire sent to selected cases and controls. Apart from dietary information, information about smoking in pregnancy was obtained. As smoking information was also part of the cohort data base, we could compare retrospective and concurrent information on smoking as presented here.

Women with language problems (461), fetal death before 28 completed weeks of gestation (120), or multiple pregnancies (259), women delivering outside of a predetermined 24 month period (590), as well as women on whom information on baseline data required for the selection procedure was missing (1657) were excluded leaving a total of 6347 women as sampling frame from which cases and controls were selected for the study.

For the case-control study, all 43 preeclamptic women and all 136 women with PIH were selected, whereas samples of 182 women of 252 with IUGR, 153 of 221 delivering preterm, and 189 of 673 delivering post-term were obtained by random sampling within each group. The control group sample were to be at least the size of the largest of the case groups. A control group of 256 women was sampled from the entire cohort ensuring that they were evenly distributed over and covering all months of the period corresponding to the recall time in the case groups. For the purpose of the present analyses women eligible for more than one case group were included in all case groups for which they were eligible. Thus, the total number of women sampled amounts to less than the sum of women in the case and control groups. A total of 1011 women were sampled for the study. 
Table 1 Measures of agreement for smoking status (smoking or not) are proportion of agreement $\left(P_{\partial}\right)$, sensitivity (sens), specificity (spef), and $\kappa$, and for number of cigarettes smoked Spearman's correlation coefficient $(r$ ) and mean difference (d). Comparison of concurrent and retrospective information on smoking habits in 2 nd half of pregnancy: subdivision according to length of recall period. (Case and control groups were aggregated). Sensitivity and specificity were estimated using concurrent information as a reference

\begin{tabular}{|c|c|c|c|c|c|c|c|c|}
\hline & \multirow[b]{2}{*}{ Number } & \multicolumn{4}{|c|}{ Smoking status (yes/no) } & \multicolumn{3}{|c|}{ Number of cigarettes smoked } \\
\hline & & $P_{0}$ & Sens & Spef & $\kappa$ & $r_{s}$ & $d$ & $95 \% C I^{\star}$ \\
\hline$<1$ year & 30 & 0.97 & 1.00 & 0.95 & 0.92 & 0.96 & -0.10 & $-0.38,0.18$ \\
\hline $1-1.5$ years & 124 & 0.97 & 0.97 & 0.97 & 0.92 & 0.95 & 0.06 & $-0.19,0.32$ \\
\hline $1.5-2$ years & 118 & 0.97 & 0.95 & 0.97 & 0.93 & 0.96 & -0.10 & $-0.50,0.29$ \\
\hline $2-2.5$ years & 132 & 0.95 & 0.97 & 0.94 & 0.87 & 0.88 & -0.01 & $-0.54,0.53$ \\
\hline $2.5-3$ years & 99 & 0.96 & 0.96 & 0.96 & 0.90 & 0.93 & -0.51 & $-0.88,-0.13$ \\
\hline
\end{tabular}

$\star 95 \%$ confidence intervals for mean difference.

Pre-eclampsia was defined as diastolic blood pressure $>90 \mathrm{~mm} \mathrm{Hg}$ with proteinuria $>0.3 \mathrm{~g} / 1$ or $>+$ using dipsticks; PIH as diastolic blood pressure $>90 \mathrm{~mm} \mathrm{Hg}$ without proteinuria; IUGR as birth weight below the 5 th centile for gestational age; preterm delivery as gestational age <259 days; and post-term delivery as gestational age $>294$ days.

For pre-eclampsia and PIH all case reports were checked manually to determine the time of diagnosis. All pre-eclamptic women and all but five women with PIH were diagnosed after 30 th week of gestation.

DATA COLLECTION METHODS

Concurrent information on smoking habits was obtained from the cohort data base and derived from a questionnaire filled in at 30th week of gestation by women attending routine antenatal care. Retrospective information from cases and controls was obtained during the autumn of 1992 from a mailed self administered questionnaire. A second set of the questionnaire was mailed in early 1993 to all non-respondents. There were slight differences in the questioning on the two occasions: Concurrent questions related to 30th week of gestation, whereas retrospective questions related to 2nd half of pregnancy. This was done to facilitate information retrieval and thus avoid reporting error. ${ }^{9}$ Questions on smoking habits were otherwise similarly phrased and related to smoking status (smoking yes/no) as well as amount smoked (number of cigarettes per day).

The concurrent questionnaire focused primarily on psychosocial factors, education and work conditions, the retrospective questionnaire on dietary habits during pregnancy. ${ }^{8}$ Thus, both questionnaires focused mainly on factors other than smoking habits.

Information on covariates was obtained from the cohort data base and included maternal alcohol intake at 30th week of gestation $(<1$, 1-2, 3-14 drinks/week), maternal height (five categories), maternal prepregnancy weight (five categories), parity (four categories), and maternal social status as defined by the Social Research Institute in Copenhagen, ${ }^{10}$ (originally five categories, of which categories 1-3 were combined because of small numbers in categories 1 and 3 ).

STATISTICAL METHODS

Various statistical measurements have been recommended for assessing validity and reli- ability of epidemiological tools. ${ }^{11-13}$ Following Bellach $^{13}$ we present the results of various statistics to reflect the stability of our results: Reproducibility of binary questions (yes/no) was assessed by proportion of agreement $\left(P_{0}\right)$ among pairwise comparison in a two by two table, sensitivity, specificity, and $\kappa$. Sensitivity and specificity were estimated using concurrent information as a reference. Reproducibility of continuous variables (number of cigarettes per day) was assessed by Spearman's correlation coefficient $\left(r_{\mathrm{s}}\right)$, and the mean difference (đ). Difference was defined as concurrent measurement-retrospective measurement.

To account for potential effect modification and confounding all analyses were stratified by maternal social status. Subsequently we performed multiple linear regression analyses using the mean difference as dependent variable. Models included as independent variables all the above covariates as well as either case status or recall time (five categories as in table 1 or as a continuous variable). Regression analyses were performed for all women together as well as for various levels of smoking.

The study design was approved by the regional ethics committee.

\section{Results}

Of the 1011 women invited to participate in the retrospective study $764(76 \%)$ returned the questionnaire with useful dietary information. Five hundred and three women $(50 \%)$ of the study base had answered all questions on smoking habits in both questionnaires. Response rates for each subgroup were $60 \%$ (preeclampsia), 57\% (PIH), 39\% (IUGR), 40\% (preterm delivery), 54\% (post-term delivery), and $65 \%$ (controls).

Proportions of smokers among respondents in each subgroup (concurrent information) were 19\% (pre-eclampsia), 9\% (PIH), 50\% (IUGR), 40\% (preterm delivery), 22\% (postterm delivery), and 26\% (controls). For comparison, proportions of smokers in each subgroup in the cohort data base (concurrent information) were 14\% (pre-eclampsia), 11\% (PIH), 55\% (IUGR), 42\% (preterm delivery), $26 \%$ (post-term delivery), and 30\% (controls).

Table 1 presents agreement between concurrent and retrospective information on smoking according to length of recall period. No substantial differences were seen in agreement as a function of recall interval, although mean differences might suggest a modest tendency towards retrospective overreporting of smoking habits. Thus, in women with the largest recall interval of 2.5-3 years, the mean difference amounted to -0.51 (95\% CI: $-0.88,-0.13)$. Apart from that the general impression was that of a high stability over time.

Table 2 presents agreement between concurrent and retrospective information on smoking according to pregnancy outcome. No noteworthy differences were seen in agreement between groups representing cases and controls.

We attempted to differentiate between various levels of smoking to evaluate whether the amount smoked influenced reliability of recall but could not detect any systematic trend in 
Table 2 Measures of agreement for smoking status (smoking or not) are proportion of agreement $\left(P_{0}\right)$, sensitivity (sens), specificity (spef), and $\kappa$, and for number of cigarettes smoked Spearman's correlation coefficient ( $r$ ) and mean difference (d). Comparison of concurrent and retrospective information on smoking habits in 2 nd half of pregnancy: subdivision according to case and control groups. Sensitivity and specificity were estimated using concurrent information as a reference

\begin{tabular}{|c|c|c|c|c|c|c|c|c|}
\hline & \multirow[b]{2}{*}{ Number } & \multicolumn{4}{|c|}{ Smoking status (yes/no) } & \multicolumn{3}{|c|}{ Number of cigarettes smoked } \\
\hline & & $P_{0}$ & Sens & Spef & $\kappa$ & $r_{s}$ & $d$ & $95 \% C I^{\star}$ \\
\hline Controls & 167 & 0.93 & 0.93 & 0.94 & 0.83 & 0.90 & -0.05 & $-0.32,0.22$ \\
\hline Pre-eclampsia & 26 & 0.92 & 1.00 & 0.90 & 0.79 & 0.88 & -0.15 & $-0.66,0.35$ \\
\hline $\mathrm{PIH}$ & 78 & 0.97 & 1.00 & 0.97 & 0.86 & 0.87 & -0.32 & $-0.84,0.20$ \\
\hline IUGR & 99 & 0.97 & 0.96 & 0.98 & 0.94 & 0.93 & -0.04 & $-0.64,0.56$ \\
\hline Preterm & 89 & 0.99 & 1.00 & 0.98 & 0.98 & 0.97 & 0.17 & $-0.32,0.65$ \\
\hline Post-term & 102 & 0.98 & 1.00 & 0.98 & 0.94 & 0.97 & -0.20 & $-0.49,0.10$ \\
\hline
\end{tabular}

$\star 95 \%$ confidence intervals for mean difference.

this data set: mean differences for women smoking $\geqslant 10$ cigarettes/day were for each recall period -0.40 (<1 year); 0.77 (1-1.5 years); 0.32 ( $1.5-2$ years); 1.72 (2-2.5 years); -2.00 (2.5-3 years), and for each case and control group 1.45 (controls); -0.50 (preeclamptic women); $\quad-0.60$ (PIH); 0.53 (IUGR); 1.24 (preterm); and -1.17 (postterm). All 95\% confidence intervals included 0 .

Stratifying analyses by social status did not change the conclusions, nor did multiple linear regression analyses, one exception being women delivering post-term and smoking $\geqslant 10$ cigarettes/day (regression coefficient $=-3.77$ $(-6.67,-0.87))$. However, for women delivering post-term and smoking 1-9 cigarettes/day the regression coefficient was $0.41(-1.90$, 2.71 ), and no systematic trend was thus apparent.

Recall of smoking habits seemed to be independently related to reported level of concurrent alcohol intake. Thus, for all women mean difference increased across alcohol categories $(-0.21,<1 \mathrm{drink} / w e e k ; 0.04,1-2$ drinks/week; $0.21,3-4$ drinks/week; 0.22, 5-14 drinks/ week), and even more so for women smoking $\geqslant 10$ cigarettes/day $(0.16 ; 0.43 ; 3.00 ; 4.33$ respectively). Among the latter subset multiple linear regression analysis, including maternal alcohol intake at 30th week of gestation (coded 1, 2, 3, 4 for each level), recall time, maternal height, maternal prepregnancy weight, parity, and maternal social status as independent variables, confirmed this result (regression coefficient for the alcohol variable $=1.31, \mathrm{p}=$ 0.003).

Non of the other potential confounding variables influenced recall.

\section{Discussion}

In these data Danish women seem to be able to accurately recall their smoking habits during pregnancy independent of recall time and pregnancy outcome. Recall, however, seems to diminish with increasing alcohol intake, particularly among women smoking $\geqslant 10$ cigarettes/day.

Previous investigations studying possible deterioration of recall over time have shown that smoking status (smoking or not) among non-pregnant women is fairly accurately recalled, ${ }^{14-16}$ whereas recall of the number of cigarettes smoked in pregnancy seems to
KEY POINTS

- Information on smoking habits during pregnancy may be accurately obtained retrospectively $0.5-3$ years in the past.

- Information on smoking habits may be accurately obtained retrospectively independent of adverse pregnancy outcomes including preterm delivery and intrauterine growth retardation.

- Accuracy of recall seems to diminish with increasing alcohol intake, particularly among women smoking $\geqslant 10$ cigarettes/ day.

diminish with time ${ }^{5}{ }^{17}$ as does recall of other life style factors such as dietary information. ${ }^{18}$ In our study the tendency towards overreporting number of cigarettes smoked after a recall period of 2.5-3 years was less than one cigarette on average, and no time trend was noted over the recall period of $0.5-2.5$ years.

Studies of possible differential misclassification have shown inconsistent results: one study ${ }^{19}$ showed no differences in accuracy of recall between cases and controls (elderly nonpregnant women), some showed slight differences that did not, however, substantially bias associations between smoking and pregnancy outcome away from the null-hypotheses, ${ }^{720}$ and some showed a clear difference, possibly because of differential misclassification. ${ }^{2122} \mathrm{We}$ found no differential misclassification, yet the results from this study may not apply to women with pregnancy outcomes perceived as more severe than those studied here, such as malformations and spontaneous abortions. ${ }^{21}$

The context in which the questions were asked may be of significance. In both questionnaires questions on smoking behaviour were mixed with a number of other questions. Smoking behaviour has thus not appeared to be the target issue. This could have reduced the tendency towards "deliberate" misreporting, which is one mechanism that may underlie recall bias. $^{23}$

Women who were willing to participate in our case-control study may have been more able and motivated to accurately recall their smoking habits than the non-respondents, making our results slightly optimistic. However, this is unlikely to put a serious limitation to the generalisability of our findings, as the proportion of smokers among respondents does not differ substantially from the proportion of smokers in the cohort.

Recall might further depend on the general acceptance of smoking in pregnancy and the emphasis placed on smoking cessation in antenatal care programmes. Smoking cessation programmes may thus increase focus in antenatal care on the adverse effects of smoking in pregnancy and hence give rise to self reproach in smokers, which subsequently may influence their recall. Smoking cessation trials were not introduced in Aarhus until $1994,{ }^{24}$ and our results may thus reflect the best potential recall of women not influenced by such self reproach. 
It is interesting to notice that women who drank 5-14 drinks/week and smoked $\geqslant 10$ cigarettes/day substantially underreported their smoking habits, on average four cigarettes/day, when asked after delivery. It is well known that women tend to decrease their alcohol consumption during pregnancy, ${ }^{25} 26$ and these women may thus represent moderate-heavy drinkers when not pregnant. Still, it is difficult to say more precisely what are the mechanisms underlying this finding. Nevertheless, the finding implies that data from studies using retrospective assessment of these exposures should be interpreted with caution.

These data add to earlier evidence ${ }^{14-16} 18$ that information on smoking habits may be obtained retrospectively in an accurate manner 0.5-3 years in the past independent of the pregnancy outcomes studied here. Accuracy, however, seems to diminish with increasing alcohol intake, particularly among women smoking $\geqslant 10$ cigarettes/day. This has potential implications for case-control studies assessing the simultaneous effects of smoking and alcohol exposure in pregnancy.

We would like to thank the staff of the Perinatal Epidemiological Research Unit for their help. Particularly, we are grateful to Tine Brink Henriksen PhD, Morten Hedegaard PhD, Professo Niels Jørgen Secher, Jannie Dalby Salvig PhD as well as Data
Manager Mr Jakob Hjort for valuable discussions and for providing data from the cohort data base.

Funding: this study was supported by The Danish Medical Funding: this study was supported by The Danish Medical Foundation.

Conflicts of interest: none.

Wilcox AJ. Birth weight and perinatal mortality: the effect of

maternal smoking. Am $\mathcal{F}$ Epidemiol 1993;137:1098-104.
2 Naeye RL. Abruptio placentae and placenta previa: frequency, perinatal mortality, and cigarette smoking. frequency, perinatal mortality,

3 Cnattingius S, Haglund B, Meirik O. Cigarette smoking as risk factor for late fetal and early neonatal death. $B M f$ 1988;297:258-61.

4 Klemetti A, Saxen L. Prospective versus retrospective approach in the search for environmental causes of malformations. Am f Public Health Nations Health 1967;57:20715.

5 Hakim RB, Tielsch JM, See LC. Agreement between maternal interview- and medical record-based gestational age. Am F Epidemiol 1992;136:566-73.

6 Golding J. Sudden infant death syndrome and maternal smoking conclusions questioned. [Letter]. Am f Public Health 1993;83:1346-7.

7 Drews CD, Kraus JF, Greenland S. Recall bias in a case-control study of sudden infant death syndrome. Int $\mathscr{f}$ Epidemiol 1990;19:405-11.
8 Kesmodel U, Olsen SF, Salvig JD. Marine n-3 fatty acid and calcium intake in relation to pregnancy induced hypertension, intrauterine growth retardation, and preterm delivery. A case-control study. Acta Obstet Gynecol Scand 1997;76:38-44.

9 Wu ML, Whittemore AS, Jung DL. Errors in reported dietary intakes. II. Long-term recall. Am f Epidemiol 1988; 128:1137-45.

10 Enevoldsen B, Michelsen N, Friis Hasche E, et al. [Social classification. II. Stalastoga's subdivision according to social status rank and the social grouping employed by the Institute for Social Research]. Sociale klassifikationer. II. Svalastoga's inddeling efter social status rangorden og socialforskningsinstituttets socialgruppeinddeling. Ugeskr Laeger 1980;142:544-50.

11 Bland JM, Altman DG. Statistical methods for assessing greement between two methods of clinical measurement. Lancet 1986;i:307-10.

12 Maclure M, Willett WC. Misinterpretation and misuse of the kappa statistic. Am f Epidemiol 1987;126:161-9.

13 Bellach B. Remarks on the use of Pearson's correlation coefficient and other association measures in assessing validity and reliability of dietary assessment methods. Eur 7 Clin Nutr 1993;47 (suppl 2):S42-5.

14 McLaughlin JK, Dietz MS, Mehl ES, et al. Reliability of surrogate information on cigarette smoking by type of informant. Am f Epidemiol 1987;126:144-6.

15 Krall EA, Valadian I, Dwyer JT, et al. Accuracy of recalled smoking data. Am f Public Health 1989;79:200-2

16 Osler M, Rasmussen NK, Lous J. [Smoking habits among adults - attitude and behavior in relation to prevention of cardiovascular diseases]. Voksnes rygevaner - holdning og adfærd i relation til forebyggelse af hjerte-kar-sygdomme. Ugeskr Laeger 1992;154:543-8.

17 Gibbons LE, Ponsonby AL, Dwyer T. A comparison of prospective and retrospective responses on sudden infant death syndrome by case and control mothers. Am F Epidemiol 1993;137:654-9.

18 Friedenreich CM, Slimani N, Riboli E. Measurement of past diet: review of previous and proposed methods. Epidemiol Rev 1992;14:177-96.

19 Paganini Hill A, Ross RK. Reliability of recall of drug usage and other health-related information. Am 7 Epidemiol 1982;116:114-22.

20 Verkerk PH, Buitendijk SE, Verloove Vanhorick SP. Differential misclassification of alcohol and cigarette consumption by pregnancy outcome. Int $\mathcal{F}$ Epidemiol 1994; 23:1218-25

21 Ericson A, Kallen B. An epidemiological study of work with video screens and pregnancy outcome: II. A case-control study. Am f Ind Med 1986;9:459-75.

22 Malloy MH, Hoffman HJ, Peterson DR. Sudden infant death syndrome and maternal smoking. Am f Public Health 1992;82:1380-2.

23 da Costa Pereira A, Olsen J, Ogston S. Variability of self reported measures of alcohol consumption: implications for the association between drinking in pregnancy and birth weight. $\mathcal{F}$ Epidemiol Community Health 1993;47:326-30.

24 Sønderskov J, Olsen J, Sabroe S, et al. Nicotine patches in smoking cessation: a randomized trial among over-thecounter customers in Denmark. Am $\mathcal{F}$ Epidemiol 1997;145: $309-18$

25 Ihlen BM, Amundsen A, Sande HA, et al. Changes in the use of intoxicants after onset of pregnancy. British fournal of Addictiction 1990;85:1627-31.

26 Passaro KT, Little RE, Savitz DA, et al. The effect of maternal drinking before conception and in early pregnancy on infant birthweight. The ALSPAC Study Team. Avon Longitudinal Study of Pregnancy and Childhood. Epidemiology 1996;7:377-83. 